\title{
Examination Under Anesthesia
}

National Cancer Institute

\section{Source}

National Cancer Institute. Examination Under Anesthesia. NCI Thesaurus. Code C40971.

A medical inspection, qualified according to the methods employed, as physical examination, radiologic examination, diagnostic imaging examination, etc., performed under conditions of patient's complete or partial local or general insensibility to pain with or without the loss of consciousness, induced by an anesthetic agent or by nonpharmacological anesthesia technique. 